Ann. Biol. anim. Bioch. Biophys., 1977, 17 (3 A), 299-307.

\title{
Effets à court et moyen terme de la ligature du canal pancréa- tique sur la digestibilité d'un aliment, chez le lapin en croissance et soumis à une alimentation restreinte.
}

\author{
par T. CORRING, F. LEBAS \\ avec la collaboration technique de Marie-Claude COUSIN et Anne-Marie GUEUGNEAU \\ Laboratoire de Physiologie de la Nutrition, \\ Laboratoire de Recherches sur l'Elevage du Lapin, \\ I.N.R.A., 78350 Jouy-en-Josas
}

Summary. Short and mean-ferm effect of pancreatic duct ligature on dief digestibility in the food-restricted growing Rabbit.

Fourteen growing rabbits are given $100 \mathrm{~g} /$ day of a diet during 4 weeks before the operation. The pancreatic duct is ligated in 8 animals $(P)$; the 6 others are sham-operated (TO). Dry matter (MS), organic matter $(M O)$, nitrogen $(N)$ and energy digestibility are measured before operation, then during 3 successive periods (2, 4 and 6 weeks) after ligature ; the animals are still given $100 \mathrm{~g} /$ day of the diet during these periods. After operation, the growth of $P$ rabbits remains lower than that of TO animals $(5.4 \mathrm{~g} /$ day versus $9.8 \mathrm{~g} /$ day) ; immediately after pancreatic duct ligature, nitrogen digestibility falls sharply (TO : $71.1 ; P: 51.9$ ). At the second post-operatory period, nitrogen digestibility increases by 3.3 points and remains constant afterwards. Energy and MS digestibilities decrease respectively by 6.4 and 4.8 points after pancreatic duct ligature. More rapid progressive improvement of energy digestibility is noted in $P$ animals, whereas the evolution of MS and MO digestibilities are comparable to those in TO animals. Finally, after the animals are killed, some measurements show that the weights of the empty caecum and of the caecal contents and the $\mathrm{pH}$ of the latter are not significantly different between $\mathrm{P}$ and TO animals. In contrast, liver weight is significantly lower in the ligated animals (liver/body weight : -16 p. 100).

\section{Introduction.}

Les recherches sur la physiologie digestive du Lapin se sont développées au cours de ces 20 dernières années (Proto et Gianani, 1969 ; Coppola, Percuoco et Proto, 1973 ; Laplace, 1975), et certains aspects particuliers de cette physiologie ont été mis en évidence : rôle du côlon (Bonnafous et Raynaud, 1967) et de la microflore intestinale (Yoshida ef al., 1968) dans la cæcotrophie, relations entre cette dernière ef le transit gastro-intestinal (Laplace et Lebas, 1975). Cependant, les rapports entre les aptitudes 
digestives du Lapin et son alimentation ne sont, en général, que très peu étudiés. Les travaux de Bacques, Demigne et Vaiton (1970), de Catala et Bonnafous (1974) laissent supposer l'importance de la sécrétion pancréatique dans l'utilisation digestive des aliments.

L'observation clinique a mis en évidence le rôle de cette sécrétion dans l'utilisafion digestive des aliments et l'absorption des nutriments dans les cas d'insuffisances pancréatiques chez l'Homme (Christensen ef Schwachman, 1949) et chez le Chien (Shingleton et al., 1955 ; Säteri, 1975). L'étude expérimentale a permis de souligner qu'après ligature du ou des canaux pancréatiques (Douglas ef al., 1953 ; Anderson ef Ash, 1971) ou après pancréatectomie totale (Shingleton et al., 1955), l'utilisation digestive et l'absorption sont plus ou moins affectées selon le composant alimentaire et selon son origine. Ainsi, chez le Porc (Anderson et Ash, 1971) après ligature du canal pancréatique, l'utilisation digestive apparente de l'azote diminue de 17 p. 100 alors que celle de l'énergie n'est pas significativement affectée.

Dans le but de préciser chez le Lapin, en croissance, le rôle du pancréas exocrine dans la digestion, nous avons réalisé une étude de la digestibilité d'un aliment après ligature du canal pancréatique, et son évolution a été suivie durant 6 semaines après l'opération.

\section{Matériel et méthodes.}

Animaux ef alimentation.

Quatorze lapins des deux sexes, de race californienne, ont été utilisés. Ils ont été placés à l'âge de 7 semaines dans des cages entièrement métalliques permettant la collecte totale des fèces.

Les animaux ont alors reçu chaque jour et en une seule fois $100 \mathrm{~g}$ d'un aliment dont la composition est donnée dans le tableau 1. L'alimentation a été restreinte de

\section{TABLEAU 1}

Composition de l'aliment expérimental

\begin{tabular}{|c|c|c|c|}
\hline Composants & p. 100 & Teneurs & p. 100 \\
\hline 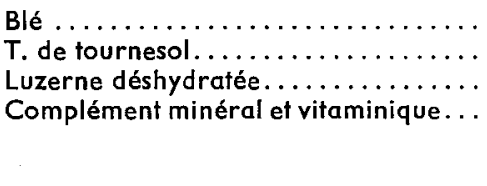 & $\begin{array}{r}37 \\
9 \\
50 \\
4 \\
100\end{array}$ & 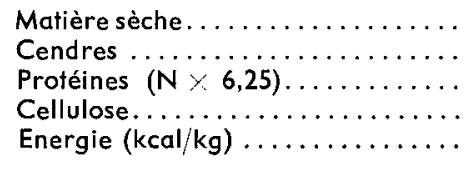 & $\begin{array}{r}89,7 \\
8,9 \\
13,8 \\
15,0 \\
3765\end{array}$ \\
\hline
\end{tabular}

manière à égaliser les ingestions de tous les animaux, ef le niveau de restriction a été maintenu constant à $100 \mathrm{~g}$ par jour durant toute l'expérimentation, afin d'éviter toute interférence entre les paramètres étudiés et le niveau d'ingestion. Par ailleurs, les 
lapins avaient à leur disposition, en permanence, un abreuvoir avec une surface d'eau libre. Enfin, les animaux ont été pesés 2 fois par semaine.

\section{Schéma expérimental.}

Après 15 jours d'adaptation des animaux à la cage et au régime alimentaire, la digestibilité de l'aliment a été mesurée pendant 4 périodes successives : une première période commençant 14 jours avant ligature du canal pancréatique, suivie de 3 périodes successives débutant respectivement à 7,21 et 35 jours après opération. Pour chacune des périodes, les collectes de fèces ont été réalisées pendant 2 fois 4 jours, réparties sur 2 semaines de calendrier (Colin et Lebas, 1976). Les coefficients d'utilisation digestive apparente $\left(C U D_{a}\right)$ de la matière sèche $(M S)$, de la matière organique $(M O)$, de l'azote $(N)$ et de l'énergie ont été mesurés selon des méthodes antérieurement décrites (Lebas, 1975).

De plus, il a été procédé au calcul du $C U_{a}$ théorique de l'énergie propre aux substances non azotées selon la formule :

$$
\text { CUD }_{\mathrm{a}}=1-\frac{(\text { Energie excrétée }- \text { Azote excrété } \times 6,25 \times 5,7)}{(\text { Energie ingérée }- \text { Azote ingéré } \times 6,25 \times 5,7)} \times 100
$$

où 5,7 est l'énergie digestible des protéines ( $\mathrm{kcal} / \mathrm{g}$ ) (Nehring, Beyer ef Hoffmann 1972).

Tous les lapins ont été abattus 46 jours après opération et il a été procédé aux mesures suivantes : poids du foie et du cæcum vide ; poids, $\mathrm{pH}$ et teneur en matière sèche du contenu cæcal.

\section{Ligature du canal pancréatique.}

Huit lapins ont subi une ligature du canal pancréatique (lot P), les 6 autres ont subi une opération fictive (lot TO).

Après $48 \mathrm{~h}$, en moyenne, de jeûne (suppression de la distribution d'aliment du jour précédant l'intervention chirurgicale) les animaux sont anesthésiés par injection intraveineuse, à l'oreille, de $10 \mathrm{ml}$ d'une solution diluée de pentobarbital sodique * ( $3 \mathrm{mg}$ principe actif $/ \mathrm{kg}$ ). Après une laparotomie médiane, l'anse duodénale est légèrement sortie de la cavité abdominale et 2 ligatures contiguës sont placées autour du canal pancréatique à $0,5 \mathrm{~cm}$ environ de son abouchement duodénal. Le canal est ensuite sectionné entre les 2 ligatures.

Les animaux opérés fictifs TO ont subi une opération identique à celle des animaux $P$, à l'exception de la pose des ligatures et de la transsection du canal pancréatique.

L'efficacité de la ligature a été contrôlée à l'abattage par les observations macroscopiques classiques : dilatation du canal pancréatique et atrophie du tissu pancréatique.

\footnotetext{
* Laboratoire Lathevet, Paris.
} 


\section{Résultats.}

\section{Croissance.}

L'évolution de la croissance des lapins TO et $\not$, durant toute l'expérimentation, est rapportée dans la figure 1. Trois périodes peuvent être distinguées :

- une première période, pré-opératoire, au cours de laquelle la vitesse de croissance est en moyenne de $26,0 \mathrm{~g}$ par jour ;

- une seconde période comprenant d'une part la mise à jeun des animaux en vue de l'intervention chirurgicale, ef d'autre part la première semaine après opération. La mise à jeun a entraîné une perte de poids de tous les animaux, tandis que la croissance est redevenue positive $(+5 \mathrm{~g} / \mathrm{j})$ durant les 8 jours après opération, et ce malgré le choc opératoire ;

- une troisième période au cours de laquelle la croissance des lapins TO a été significativement supérieure $(11,8 \mathrm{~g} / \mathrm{j} ; \mathrm{P}<0,05)$ à celle des animaux $\not{P}(7,5 \mathrm{~g} / \mathrm{j})$. Par ailleurs, la consommation de l'aliment a diminué durant les premiers jours qui ont suivi l'intervention chirurgicale ef elle est redevenue totale pour tous les animaux dès le quatrième jour post-opératoire.

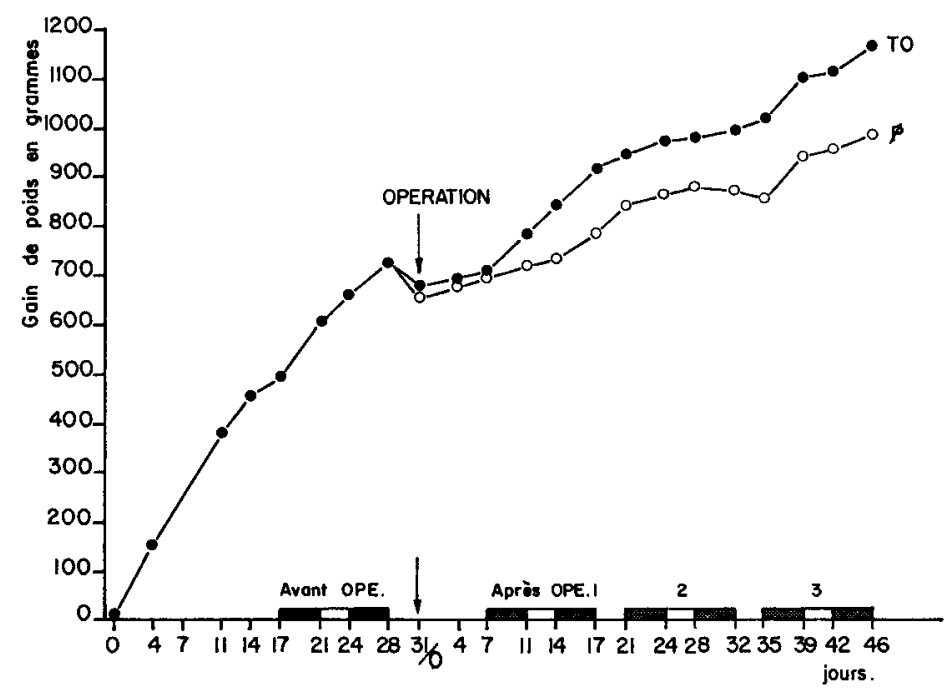

FIG. 1. - Evolution des gains de poids cumulés des lapins fot to au cours de l'expérimentation.

$\because \because$ : périodes de collectes des fèces.

- $\$$ = lapins ligaturés.

$\mathrm{O}-\mathrm{O}:$ TO = lapins témoins.

Enfin, on peut constater une évolution identique de la croissance des animaux $\$$ et TO au cours de la première semaine post-opératoire. C'est à partir de la fin de cette dernière que les courbes de croissance commencent à diverger. 


\section{Digestibilité.}

Les divers coefficients d'utilisation digestive apparente $\left(C \mathrm{CD}_{\mathrm{a}}\right)$ déterminés au cours de l'expérience sont rapportés dans le tableau 2.

Il apparaît que la ligature du canal pancréatique, chez le Lapin en croissance, affecte principalement le CUD $D_{a}$ de l'azote : celui-ci subit une diminution de 19,8 points au cours de la première période de collecte post-opératoire, par rapport aux valeurs observées pour les lapins TO. Par contre, au cours de la période suivante, on enregistre une amélioration de sa valeur puisque la digestibilité apparente de l'azote augmente entre ces 2 périodes post-opératoires de 3,3 points. Elle reste stable par la suite.

TABLEAU 2

Evolution des coefficients de digestibilité de l'aliment chez les lapins TO ef $P$ avant et après opération

\begin{tabular}{|c|c|c|c|c|c|c|}
\hline \multirow{3}{*}{\multicolumn{2}{|c|}{$\mathrm{CUD}_{\mathrm{a}}$}} & \multicolumn{5}{|c|}{ Périodes } \\
\hline & & \multirow{2}{*}{$\begin{array}{c}\text { avant } \\
\text { opération }\end{array}$} & \multicolumn{4}{|c|}{ après opération } \\
\hline & & & 1 & & 2 & 3 \\
\hline Azote & $\begin{array}{l}\text { TO } \\
\mathrm{P}\end{array}$ & $\begin{array}{l}68,2^{\mathrm{a}} \pm 2,2\left({ }^{1}\right) \\
69,2^{\mathrm{a}} \pm 1,6\end{array}$ & $\begin{array}{r}71,7 \mathrm{a} \\
\left({ }^{2}\right)^{* * * *} 51,9 \mathrm{~b} \\
\end{array}$ & $\begin{array}{l} \pm 1,3 \\
\pm 2,1 \\
\end{array}$ & $\begin{array}{r}70,9 \mathrm{a} \\
* 0,7 \\
* * * 55,2^{\mathrm{c}} \pm 1,4 \\
\end{array}$ & $\begin{array}{r}70,5^{\mathrm{a}} \pm 1,5 \\
* * * 55,4^{\mathrm{c}} \pm 1,8 \\
\end{array}$ \\
\hline MS & $\begin{array}{l}\text { TO } \\
\mathrm{P} \\
\end{array}$ & $\begin{array}{l}64,0^{\mathrm{a}} \pm 0,7 \\
65,2^{\mathrm{a}} \pm 0,7 \\
\end{array}$ & $\begin{array}{r}65,6 \mathrm{~b} \\
* * 60,8^{b} \\
\end{array}$ & $\begin{array}{r} \pm 0,6 \\
\pm 1,0 \\
\end{array}$ & $\begin{array}{r}66,9 \mathrm{bc} \pm 0,3 \\
* * * 61,5 \mathrm{bc} \pm 1,2 \\
\end{array}$ & $\begin{array}{r}67,7^{\mathrm{c}} \pm 1,0 \\
* 64,2^{\mathrm{c}} \pm 0,7 \\
\end{array}$ \\
\hline MO & $\begin{array}{l}\text { TO } \\
\mathrm{P}\end{array}$ & $\begin{array}{l}64,8 \mathrm{a} \pm 0,7 \\
66,1^{\mathrm{a}} \pm 0,7\end{array}$ & $\begin{array}{r}66,6^{\mathrm{b}} \\
* * 61,7^{\mathrm{b}} \\
\end{array}$ & $\begin{array}{r} \pm 0,6 \\
\pm 1,0 \\
\end{array}$ & $\begin{array}{r}68,4 \mathrm{bc} \pm 0,1 \\
* * * 62,9 \mathrm{bc} \pm 1,1 \\
\end{array}$ & $\begin{array}{r}69,3 \mathrm{c} \pm 1,0 \\
* 65,7 \mathrm{c} \pm 0,7 \\
\end{array}$ \\
\hline Energie & $\begin{array}{l}\text { TO } \\
\mathrm{P}\end{array}$ & $\begin{array}{l}62,4^{\mathrm{a}} \pm 0,8 \\
64,0^{\mathrm{a}} \pm 0,9 \\
\end{array}$ & $\begin{array}{r}64,7 \mathrm{ab} \\
* * 58,3^{b} \\
\end{array}$ & $\begin{array}{l} \pm 0,9 \\
\pm 1,0\end{array}$ & $\begin{array}{r}65,6^{\mathrm{bc}} \pm 0,4 \\
* * 59,8^{\mathrm{c}} \pm 1,4 \\
\end{array}$ & $\begin{array}{r}66,7^{\mathrm{c}} \pm 0,9 \\
* * 62,8^{\mathrm{a}} \pm 0,9 \\
\end{array}$ \\
\hline Energie- $\mathrm{N}$ & $\begin{array}{l}\text { TO } \\
P\end{array}$ & $\begin{array}{l}62,5^{\mathrm{a}} \pm 0,8 \\
63,9^{\mathrm{a}} \pm 0,9\end{array}$ & $\begin{array}{r}64,1^{\mathrm{ab}} \\
* * 58,4^{\mathrm{b}}\end{array}$ & $\begin{array}{l} \pm 1,1 \\
\pm 1,0\end{array}$ & $\begin{array}{r}65,4^{\mathrm{bc}} \pm 0,4 \\
* * 60,4^{\mathrm{c}} \pm 1,4\end{array}$ & $\begin{array}{r}66,6^{c} \pm 0,8 \\
* 62,9^{a} \pm 0,9\end{array}$ \\
\hline
\end{tabular}

(1) Moyenne \pm écart type de la moyenne.

( ${ }^{2}$ Les $C U D_{a}$ pour $P$ el TO diffèrent au seuil : * $P=0,05 ; * * P=0,01 ; * * * P=0,001$

$a, b$... Sur une même ligne, les valeurs ayant la même lettre en indice ne sont pas significativement différentes au seuil $P=0,05$.

MS : matière sèche; $M O$ : matière organique ; Energie- $N$ : Energie provenant des substances non azotées.

En ce qui concerne les $C U D_{a}$ de la matière sèche et de l'énergie, la ligature du canal pancréatique entraîne (par rapport aux animaux TO) au cours de la première période post-opération, une légère réduction de la digestibilité de la $M S$ (-4,8 points) et de celle de l'énergie (-6,4 points). On constate par la suite une augmentation générale des valeurs des $C U D_{a} M S$ et énergie ( $P$ ef TO). Cependant, cette augmentation est significativement plus rapide pour l'énergie chez les animaux $P(+4,5$ points entre les périodes 1 et 3 , vs 1,1 points chez les animaux $T O$ ), et elle est non significativement différente entre $P(+3,3$ points) ef TO $(2,2$ points) pour la matière sèche. 
Enfin, l'évolution du CUDa de la matière organique est semblable à celle du CUDa de la matière sèche.

Si l'on considère à présent l'évolution des CUD $D_{a}$ de l'énergie due aux composants non azotés du régime (principalement les glucides), on constate que les valeurs calculées sont significativement inférieures chez les animaux $\mathbf{P}$ au cours des 3 périodes post-opératoires. Par ailleurs, on note une amélioration des valeurs de la 1 re à la $3^{\text {e }}$ période après opération aussi bien chez les animaux $\mathrm{P}(+4,5$ points) que chez les animaux TO $(+2,5$ points $)$.

Mesure après abatfage des animaux.

Les poids du foie, du cæcum vide et du contenu cæcal ainsi que le $\mathrm{pH}$ de ce dernier, chez les animaux $\mathrm{P}$ et TO après abattage, sont rapportés dans le tableau 3.

\section{TABLEAU 3}

Résultats après abattage des animoux

\begin{tabular}{|c|c|c|c|}
\hline \multirow{2}{*}{ Paramètres mesurés } & \multicolumn{2}{|c|}{ Traitement } & \multirow{2}{*}{$\begin{array}{l}\text { Signification } \\
\text { statistique } \\
\text { (t de Student) }\left({ }^{1}\right)\end{array}$} \\
\hline & TO & $\mathbf{P}$ & \\
\hline Poids du foie $\left\{\begin{array}{l}\text { grammes } \ldots . . . \ldots \\
\text { p. } 100 \text { poids vif...... }\end{array}\right.$ & $\begin{array}{l}67,3 \pm 3,0 \\
2,92 \pm 0,11\end{array}$ & $\begin{aligned} 51,6 & \pm 2,3 \\
2,44 & \pm 0,19\end{aligned}$ & $\begin{array}{l}4,26 * * \\
3,15 * *\end{array}$ \\
\hline Poids du cæcum . . . . . . . . . . . . . & $52,3 \pm 1,0$ & $51,6 \pm 4,0$ & $0,16 \mathrm{NS}$ \\
\hline $\begin{array}{c}\text { Contenu } \\
\text { cæcal }\end{array}\left\{\begin{array}{l}\text { poids frais }(g) \ldots \ldots \ldots \ldots \\
\text { poids sec }(\mathrm{g}) \ldots \ldots \ldots \ldots \ldots \\
\text { pH } \ldots \ldots \ldots \ldots \ldots \ldots \ldots\end{array}\right.$ & $\begin{array}{c}167 \pm 9,0 \\
38,7 \pm 2,2 \\
6,02 \pm 0,27\end{array}$ & $\begin{array}{l}174 \pm 16,0 \\
37,1 \pm 3,9 \\
6,02 \pm 0,25\end{array}$ & $\begin{array}{l}0,31 \mathrm{NS} \\
0,33 \mathrm{NS} \\
0,01 \mathrm{NS}\end{array}$ \\
\hline
\end{tabular}

(1) $* * P=0.01 ; \mathrm{NS}=$ non significatif.

Il apparaît que tous les critères concernant le cæcum ne sont pas significativement différents entre les animaux $P$ et TO. Par contre, le poids du foie est significativement réduit $(P<0,01)$ chez les animaux $P$ par rapport à celui des lapins TO, qu'il soit exprimé en valeur absolue ( -23 p. 100) ou en fonction du poids vif ( -16 p. 100).

\section{Discussion,}

La ligafure du canal pancréatique chez le Lapin en croissance et soumis à une alimentation restreinte, se traduit dans les 3 semaines qui suivent, par une modification de l'utilisation digestive de l'aliment ingéré. C'est l'utilisation digestive de l'azote alimentaire qui est essentiellement affectée puisque le $C U D_{a}$ correspondant diminue en moyenne de 27,6 p. 100.

Les $C U D_{a}$ de l'énergie diminuent également mais dans des proportions moindres (en moyenne : - 7,3 p. 100 pour la matière sèche et la matière organique et $-9,9$ p. 100 pour l'énergie). 
L'effet de la suppression de l'hydrolyse enzymatique pancréatique des protéines alimentaires sur leur utilisation digestive a été mis en évidence chez d'autres espèces. Cependant la diminution du CUD $\mathrm{C}_{\mathrm{a}}$ correspondante est en général inférieure à celle enregistrée chez le Lapin, dans cette expérience. Ainsi chez le Porc olle est de 17 p. 100 après ligature du canal pancréatique selon Anderson et Ash (1971) et de 13 p. 100 au cours des 5 journées qui suivent la dérivation de la sécrétion pancréatique après fistulation selon Corring et Bourdon (1976a). Chez le Rat, elle est de l'ordre de 22 p. 100 (Uram, Friedman et Kline, 1960) ou 17 p. 100 (Clowes et MacPherson, 1951). Ces différences sont vraisemblablement dues en partie aux caractères physiologiques propres à chaque espèce mais également à la nature des composants alimentaires ingérés par les sujets de ces diverses espèces. Ainsi Uram, Friedman et Kline (1960) montrent que le Rat privé de sa sécrétion pancréatique exocrine utilise mieux l'azote des protéines d'origine animale que celui des protéines d'origine végétale.

En ce qui concerne le $C U D_{a}$ de l'énergie, les résultats chez le Lapin confirment ceux obtenus chez le Porc (Anderson et Ash, 1971 ; Corring et Bourdon, 1976a). Chez cette espèce, la suppression de l'hydrolyse enzymatique pancréatique n'affecte que très légèrement la digestibilité de l'énergie. Dans le régime expérimental utilisé dans la présente expérience, les lipides ne représentent que 3 p. 100 de la matière sèche alors que les glucides en représentent 71 p. 100. Ces derniers sont donc la principale source d'énergie alimentaire. La réduction du $\mathrm{CUD}_{\mathrm{a}}$ de la fraction EnergieAzote (énergie provenant des substances non azotées : lipides + glucides) est donc essentiellement la conséquence de la suppression de l'hydrolyse pancréatique sur la digestibilité des glucides. L'amidon représentant environ 35 p. 100 des glucides fotaux, on peut supposer que la diminution de la digastibilité de la fraction Energie-Azote est en partie due à une digestion réduite de cet élément, consécutive à l'absence d'amylase pancréatique.

Il est intéressant de suivre l'évolution des divers $C U D_{a}$ après ligature du canal pancréatique. On constate une amélioration, avec le temps, des différentes valeurs. Cette amélioration traduit la mise en place progressive d'une compensation digestive. Ainsi, chez les animaux ligaturés, entre la $1^{\text {re }}$ et la 3 e période post-opératoire, les CUD $_{a}$ de la matière sèche, matière organique, azote ef énergie ont subi une augmentation (respectivement $+5,30,+6,10$, $+6,30$ et $+7,20$ p. 100) significativement supérieure à celle des $C \mathrm{DD}_{\mathrm{a}}$ chez les animaux témoins. L'existence d'une compensation digestive après ligature du canal pancréatique a également été soulignée chez d'autres espèces. Ainsi chez le Rat (Uram, Friedman et Kline, 1960) la digestibilité apparente des protéines de céréales est améliorée de $32 \mathrm{p}$. 100 environ entre le $3^{\mathrm{e}}$ et $40^{\mathrm{e}}$ jour post-opération. Chez le très jeune Porcelet (Pekas, Hays et Thompson, 1964) la digestibilité apparente des protéines du tourteau de soja est améliorée de 32 p. 100 environ entre la $2^{\mathrm{e}}$ et $8^{\mathrm{e}}$ semaine d'âge. L'origine de cette compensation digestive n'est pas connue. En ce qui concerne l'utilisation digestive de l'énergie, on peut supposer que l'exclusion de la sécrétion pancréatique de la lumière intestinale a entraîné, entre autres, soit une stimulation des amylases salivaire et intestinale, soit l'établissement d'une compensation digestive d'origine bactérienne (Catala ef Bonnafous, 1974). En ce qui concerne l'utilisation digestive des protéines, des résultats obtenus chez le Porc (Corring et Bourdon, 1976b) conduisent à penser que la compensation digestive serait due à une hypersécrétion des pepsines gastriques. 
La croissance des lapins privés de la sécrétion pancréałique exocrine a été significativement inférieure à celle des animaux témoins, mais elle est demeurée positive dès le $4^{e}$ jour après opération. Ce dernier résultat est opposé à celui obtenu par Lepkovsky, Nalbandov et Dimick (1964) et par Clowes ef MacPherson (1951) qui signalent, respectivement chez le Poulet et le Rat, une perte de poids après ligature du canal pancréatique. Dans la présente expérience, on peut constater que la vitesse de croissance des lapins témoins a été inférieure au cours des 3 dernières semaines postopératoires à celle enregistrée avant opération. Cela s'explique par le niveau d'alimentation constant $(100 \mathrm{~g} / \mathrm{j})$ adopté pour la durée totale de l'expérimentation, qui entraîne une réduction progressive de l'apport alimentaire par rapporł au poids vif.

Les variations de digestibilité, et particulièrement celle de l'azote, ont conduit à supposer qu'une plus grande quantité d'ingesta parvenait dans le cæcum des lapins ligaturés par rapport à celle arrivant dans le cæcum des lapins témoins, ef que de ce fait, 46 jours après l'opération, le cæcum pouvait avoir subi une hypertrophie. Les mesures effectuées après abattage, montrent qu'il n'en est rien puisque le poids du cæcum vide est identique chez les animaux ligaturés et témoins ; il en est de même du poids du contenu cæcal. Par ailleurs, le $\mathrm{pH}$ de ce dernier est identique dans les 2 lots d'animaux, ce qui confirme et complète les observations de Catala (1975) sur la même espèce. Cet auteur montre en effet qu'après ligature du canal pancréatique, le $\mathrm{pH}$ mesuré dans les parties distales de l'intestin grêle est semblable à celui enregistré chez les animaux témoins, alors qu'il diffère sensiblement au niveau de l'estomac et du duodénum.

Enfin, il est intéressant de souligner la diminution du poids relatif du foie chez les Lapins, après ligature du canal pancréatique (-16 p. 100). Cela pourrait s'expliquer en partie par la réduction quantitative de l'apport des nutriments d'origine digestive, ce qui entraînerait une diminution de la fonction de stockage du foie. La réduction d'absorption due à la diminution de l'utilisation digestive après suppression de l'hydrolyse enzymatique pancréatique peut être en effet assimilée à une déplétion alimentaire protéique et il a été montré que dans ce dernier cas, chez le Porc (Nielsen, 1973) ef chez le Rat (Montecuccoli, Novello et Stirpe, 1972), le poids du foie est réduit.

Accepté en novembre 1976.

\section{Références}

ANDERSON D. M., ASH R. W., 1971. The effect of ligating the pancreatic duct on digestion in the pig. Proc. Nutr. Soc., 30, 34 A-35 A.

BACQUES C., DEMIGNE C., VAITON C., 1970. Influence de la ligature du canal pancréatique ef de la cæcumectomie sur l'excrétion fécale des lipides chez le lapin. C. R. Soc. Biol. Fr., 164, 1500-1504.

BONNAFOUS R., RAYNAUD P., 1967. Recherches sur le rôle du côlon dans la dualité d'excrétion fécale du lapin. Arch. Sci. Physiol., 21, 261-270.

CATALA J., BONNAFOUS R., 1974. Variations quantitatives de la microflore intestinale du lapin après ligature du canal pancréatique. C. R. Acad. Sci., Ser. D, 278, 3351-3353.

CATALA J., 1975. Effet de la ligature du canal pancréatique sur le $\mathrm{pH}$ intestinal chez le lapin. $C . R$. Acad. Sci., Série D, 281, 1991-1994. 
CHRISTENSEN H. N., SCHWACHMAN H., 1949. Determination of the plasma glycemia after gelatin feeding as a diagnostic procedure for pancreatic fibrosis. J. Clin. Invest., 28, 319-321.

CLOWES G. H. A. Jr., MCPHERSON L. B., 1951. Production of fatty livers by ligature of the pancreatic duct in Rats. Am. J. Physiol., 165, 628-638.

COLIN M., LEBAS F., 1976. Méthodes d'étude de la digestibilité des aliments chez le lapin. II. Périodicité des récoltes. Sci. Tech. anim. Lab., 1, 129-133.

COPPOLA S., PERCUOCO G., PROTO V., 1973. Qualche aspetto del metabolismo della microflora ciecale nella nutrizione proteica del coniglio. Boll. Soc. It. Biol. Sper., 49, 1253-1259.

CORRING T., BOURDON D., 1976a. Short-dated influence of removal of the exocrine pancreatic secretion (enzymatic or total) upon the apparent digestibility of a diet in the pig. Nutr. Rep. Int., 14, 621-627.

CORRING T., BOURDON D., 1976b. Données non publiées.

DOUGLAS G. J., REINAUER A. J., BROOKS W. C., PRATT J. H., 1953. The effect on digestion and absorption of excluding the pancreatic juice from the intestine. Gostroenterology, 23, 452-459.

LAPLACE J. P., 1975. Le transit digestif chez les monogastriques. II. Phénomènes moteurs et mouvements des digesta. Ann. Zootech., 24, 489-552.

LAPLACE J. P., LEBAS F., 1975. Le transit digestif chez le lapin. III. Influence de l'heure et du mode d'administration sur l'excrétion fécale du Cérium 141 chez le lapin alimenté od libitum. Ann Zootech., 24, 255-265.

LEBAS F., 1975. Influence de la teneur en énergie de l'aliment sur les performances de croissance chez le lapin. Ann. Zootech., 24, 281-288.

LEPKOVSKY S., NALBANDOV A. V., DIMICK M. K., 1964. Growth and reproduction of depancreatized chickens. Endocrinology, 74, 207-221.

MONTECUCCOLI G., NOVELLO F., STIRPE F., 1972. Effect of protein deprivation and of starvation on DNA synthesis in resting and regenerating rat liver. J. Nutr., 102, 507-514.

NEHRING K., BEYER M., HOFFMANN B., 1972. Futtermitfel tabellenwerk. Veb Deutscher Landwirtschaftsverlag Berlin p. 261.

NIELSEN H. E., 1973. Growth and development in pigs in the pre- and postnatal period with special reference to lafer growth and carcass composition. Beretning fra fors gslaboratoriet Kobenhavn.

PEKAS J. C., HAYS V. W.. THOMPSON A. M., 1964. Exclusion of the exocrine pancreatic secretion. Effect on digestibility of soybean and milk protein by baby pigs at various ages. J. Nutr., 82, 277-286.

PROTO V., GIANANI L., 1969. La composizione aminoacidica di alcuni alimenti delle feci e del ciecotrofo nel coniglio sottoposto a differente diele. Prod. anim., 8, 203-208.

SATERI H., 1975. Investigations of the exocrine pancreatic function in dogs suffering from chronic exocrine pancreatic insufficiency. Acta Vet. Scand., supp. 53, 1-86.

SHINGLETON W. W.. WELLS M. H., BAYLIN G. H., RUFFIN J. M., SAUNDERS A., DURHAM N. C., 1955. The use of radioactive labelled protein and fat in the evaluation of pancreatic disorders. Surgery, 38, 134-142.

URAM J. A., FRIEDMAN L., KLINE O. L., 1960. Relation of pancreatic exocrine to nutrition of the rat. Am. J. Physiol., 199, 387-394.

YOSHIDA T., PLEASANTS J. R., REDDY B. S., WOSTMANN B. S., 1968. Efficiency of digestion in germ-free and conventional rabbits. Brit. J. Nutr., 22, 723-737. 